\title{
PAH-RELATED VERY SMALL GRAINS IN PHOTODISSOCIATION REGIONS: IMPLICATIONS FROM MOLECULAR SIMULATIONS
}

\author{
M. Rapacioli ${ }^{1}$, F. Spiegelman ${ }^{1}$, B. Joalland ${ }^{1,2}$, A. Simon ${ }^{1}$, \\ A. Mirtschink ${ }^{1}$, C. Joblin ${ }^{2}$, J. Montillaud ${ }^{2}$, O. Berné ${ }^{3}$ and D. Talbi ${ }^{4}$
}

\begin{abstract}
The analysis of mid-IR emission suggests that a population of PAH-related very small grains containing a few hundreds of atoms are present in the deep regions of molecular clouds, although no specific species has been identified yet. In this review, we discuss several candidates for these grains: neutral and ionised PAH clusters and complexes of PAHs with $\mathrm{Si}$ atoms. The theoretical modelling of the properties of such molecular complexes or nanograins is a challenging task. We first present an overview of quantum chemistry derived models which can be efficiently used on-the-fly in extensive sampling of the potential energy surfaces, as required by structural optimization, classical molecular dynamics or Monte Carlo algorithms. From the simulations, various observables can be determined, such as the binding energies, finite temperature IR spectra, nucleation and evaporation rates. We discuss the relevance of those candidates in the molecular clouds photodissociation regions and propose constrains and perspectives for the nature and size of those very small grains.
\end{abstract}

\section{Introduction}

Polycyclic Aromatic Hydrocarbons (PAHs) are usually considered to be at the origin of the Aromatic Infrared Bands (AIBs) observed in emission from UV-irradiated interstellar matter (Allamandola et al. 1985; Léger \& Puget 1984,

\footnotetext{
${ }^{1}$ Laboratoire de Chimie et Physique Quantiques, Université de Toulouse (UPS) and CNRS, IRSAMC, 118 route de Narbonne, 31062 Toulouse, France

2 Université de Toulouse, UPS, CESR, 9 avenue du colonel Roche, 31062 Toulouse Cedex 4, France and CNRS, UMR 5187, 31028 Toulouse, France

3 Leiden Observatory, Leiden University, Niels Bohrweg 2, 2333 CA Leiden, The Netherlands

4 Université Montpellier II - GRAAL, CNRS - UMR 5024, Place Eugène Bataillon, 34095 Montpellier, France
} 
see also Peeters, this volume). Boulanger et al. (1990), Bernard et al. (1993) analysed the emission in the IRAS photometric bands measured for several molecular clouds, and suggested that free-flying PAHs are produced by photoevaporation of larger grains. The observation of a mid-IR continuum in the reflection nebula Ced 201 has been attributed by Cesarsky et al. (2000) to Very Small carbonaceous Grains (VSGs) that have been proposed as precursors of the AIB carriers.

Blind signal separation methods like non-negative matrix factorisation or singular value decomposition (combined with a Monte Carlo search of physical solutions) have been applied to extract three characteristic spectra from mid-IR spectro-imagery data (ISO and Spitzer) of the PhotoDissociation Region (PDR) NGC 7023 (Berné et al. 2007; Rapacioli et al. 2005, see Berné, this volume). Two spectra have been assigned to PAH populations dominated either by neutral or ionised molecules. The third spectrum is composed of broader AIBs associated with a continuum. As these two characteristic features suggest larger systems, this component has been attributed to a population of PAH-related VSGs. The spatial distribution of these spectra shows that the ionised PAH population is located around the star followed by the neutral PAH population and then by the VSG population when going further from the star and inside the molecular cloud. This suggests a chemical scenario in which PAHs are trapped in VSGs that are photoevaporated at the border of the cloud where the UV flux increases. Closer to the star, the UV flux is strong enough to keep the PAH population mainly positively ionised.

The presence of VSGs related to PAHs has been reported in several PDRs (NGC 7023, $\rho$ Ophiuchi, Ced 201; Berné et al. 2007) but also in protoplanetary disks (Berné et al. 2009) and in planetary nebulae both in the Milky Way and in the Magellanic clouds (Joblin et al. 2008).

A minimal size of these grains can be estimated to 400 carbon atoms from the grain heating process as described in Rapacioli et al. (2006). In this paper, we review different hypotheses for PAH-related VSGs and show the capability of theoretical modelling as a tool to investigate these clusters. Three possible species are mostly discussed here: neutral and ionised PAH clusters and complexes of PAHs with Si atoms.

\section{Possible candidates for the PAH-related VSGs}

Several hypothesis can be made for the PAH-related VSGs. Possible candidates that will be discussed in the following are:

1) Neutral PAH clusters: based on the sequential evolution $\mathrm{PAH}^{+}, \mathrm{PAH}^{0}$, VSG that is observed in molecular clouds, the most intuitive candidates are PAH clusters. As these clusters are located in deeper regions of molecular clouds than the neutral PAH population, it seems consistent to investigate neutral PAH clusters. 2) Ionised PAH clusters: as will be discussed in Section 5, the lowering of the ionisation potential (IP) in PAH clusters compared to PAH could allow the presence of ionised PAH clusters deeper inside molecular clouds. This hypothesis is reinforced because -(i)- recent calculations (Rhee et al. 2007) show that small closed-shell 
PAH cationic dimers could be at the origin of the Extended Red Emission (ERE) and -(ii)- in NGC $7023 \mathrm{~N}$, the ERE spatially correlates with the maximum of VSG emission (Berné et al. 2008).

3) Complexes of PAHs with an heteroatom: complexes with Fe atoms can form easily from energetics considerations and have recently been studied both experimentally and theoretically (Simon \& Joblin 2007, 2009, 2010; Simon et al. 2008) and are the subject of a specific contribution (Simon et al.) in this volume. In a similar way, complexes of PAH molecules or clusters with Si atoms could be considered as candidates for the VSGs and are presented in the last section.

This list is of course not exhaustive and the VSGs are most probably made of mixture of various types of grains rather than belonging entirely to one of the previously enumerated families.

\section{Theoretical methods adapted to large systems}

In the following, we present specific methods that we have used with some theoretical development when needed. The treatment of large clusters containing a few hundreds of atoms is a challenging task for theoretical chemistry. The Density Functional Theory (DFT) is the most widely used method to describe electronic structure, based on a density spanned on molecular orbitals and the solution of a mean-field type single electron Kohn-Sham equation. However, despite fast algorithmic developments, it can presently hardly treat such large clusters, in particular as soon as global Potential Energy Surface (PES) exploration or molecular dynamics (MD) are needed. An alternative for even larger systems and/or larger computational efficiency is provided by approximate schemes of DFT, for instance the Density Functional based Tight-Binding approach (DFTB, Elstner et al. 1998; Oliveira et al. 2009; Porezag et al. 1995; Seifert et al. 1996). DFTB is based on a second order Taylor expansion of the DFT energy expression around a reference electronic density and the expression of the second order term as a function of atomic charges. The molecular orbitals are spanned in a minimal set of valence atomic orbitals. All integrals are parametrised and the ground state is found by solving self-consistently the approximate Kohn-Sham secular equation (Self-Consistent-Charge DFTB), including electrostatics self-consistently. In principle, DFTB parametrisation is extracted from DFT calculations on the various atom-pairs and may be expected to show satisfactory transferability. Nonetheless, several parametrisations do exist, adapted to various situations (bulk, biological systems). Parametrisation adjustment may be sometimes needed. In further approximations, the self-consistency can be circumvented, or the electrostatics totally ignored, leading to simple Tight-Binding (TB) schemes. At the present time, DFT (with the most common functionals) and DFTB experience theoretical difficulties such as (i) their failure to describe long-range dispersion forces; (ii) the failure to describe dissociation properly partly screened in the spin-unrestricted formulation; (iii) the difficulty to describe properly open-shell system and multiplets; (iv) the difficulty of todays functionals in the Kohn-Sham formalism to properly describe systems with strong multi-configurational character. This has 
motivated the search for new functionals and also ab initio hybrid methods based on both the wavefunction for long-range electron-electron correlation and DFT for the short-range correlation (Chabbal et al. 2010; Fromager \& Jensen 2008; Toulouse et al. 2004). The lack of dispersion can also be corrected by addition of empirical atom-atom dispersion potentials in both DFT (Grimme 2006) and DFTB schemes (Elstner et al. 2001; Rapacioli et al. 2009; Zhechkov et al. 2005).

Moreover, in the case of molecular clusters, one can benefit from the partition of the cluster into identifiable units to propose further modelling with different levels of treatments for intra- and intermolecular degrees of freedom. This gives rises to a partition of the level of treatment of the degrees of freedom such as for instance in the case of neutrals: (i) a hybrid approach in which the molecular units (not rigid) are treated via DFT, DFTB or TB (actually developed, see below) and the intermolecular interactions (repulsion, electrostatics and dispersion) are treated via atom-atom classical potentials. This provides a consistent description between intra-molecular charge variations and intermolecular electrostatics. Such model can be considered as a special version of quantum-mechanics/molecular (classical) mechanics (QM-MM, i.e. DFTB-MM) schemes. (ii) a more basic rigid molecule approximation (for a review see Stone 1997), where the molecules constituting a cluster are treated as rigid frozen systems and only the intermolecular potential is considered. The rigid body approach obviously is the fastest one and allows global exploration of the intermolecular PES for quite large systems with hundreds of atoms. The advantage of the hybrid (DF)TB/MM approach is its ability to describe energy transfers during collisions or couplings between interand intramolecular vibrational modes.

The molecular partition is also relevant for resonance in ionised molecular clusters. Specific approaches consist in expressing the multiconfigurational wavefunction of the ionized cluster as an expansion of charge-localised configurations (Wu et al. 2007), A small Configuration Interaction matrix (CI) is then built in this limited valence-bond-like multiconfigurational basis. Such DFT-CI model is clearly very well suited to model charged molecular clusters. It can even be made more efficient in the scope of large systems when using DFTB instead of DFT: benchmark calculations on the benzene, coronene and water dimers show that the DFTB-CI method (Rapacioli et al. 2010) is able to correct the wrong behavior of the DFTB dissociation pathway and gives binding energies in agreement with experimental data and reference calculations.

\section{Neutral PAH clusters}

\subsection{Structural properties}

\subsubsection{PAH dimers}

The intermolecular interactions vanish quickly with increasing distance, except for the electrostatic terms which essentially account for interactions between permanent multipoles. The structural growth patterns of PAH clusters are therefore closely 


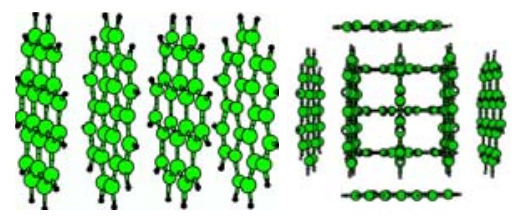

Fig. 1. Stacked coronene clusters of 4 and 10 molecules.

related to the dimer structures. Stacks are expected if the most stable dimer structures have sandwich-like configurations. The structures of PAH dimers ranging from benzene to coronene have been optimized at different levels of theory in recent years. The structure of the benzene dimer has been a long-standing discussion until high-level calculations (CCSD(T), SAPT see for instance Podeszwa et al. 2006) showed that the PES of this dimer has several almost degenerated structures corresponding to T-shaped and sandwich-parallel-displaced geometries. The stacked sandwiches are less stable. For larger PAHs, the T-shaped configurations are strongly destabilised as compared to sandwich structures both in parallel-displaced and stacked configurations. The binding energy increases from about $0.12 \mathrm{eV}$ for the benzene dimer to $0.8-1 \mathrm{eV}$ for the coronene dimer (Rapacioli et al. 2009; Zhao \& Truhlar 2008). The rigid body, TB-MM and DFTB approaches have been parametrised and benchmarked (Rapacioli et al. 2005, 2006, 2009) on these high-level calculations on PAH dimers.

\subsubsection{Large coronene clusters}

A global exploration of the PES using parallel tempering Monte-Carlo methods with the rigid body approach provides the structures of coronene clusters containing up to 32 molecules (Rapacioli et al. 2005). As expected from the dimer sandwich structures, the lowest-energy structures of coronene clusters are found to be single stacks for numbers of molecules not exceeding 8. For larger clusters, lower energy structures appear made of two stacks with either parallel axis or in a handshake-like structure (axes almost perpendicular, Fig. 1). The special stability of the "handshake" structure is due to its compactness, while conserving the primary stack structure as basic units. The $(\mathrm{PAH})_{32}$ cluster presents a large number of degenerated isomers which all consist of small units of 4 molecule stacks aggregated to each others. The dissociation energy of these clusters (energy required to remove one molecule) is almost constant (about $1 \mathrm{eV}$ ) for clusters above 3 molecules as the less bounded molecules are always at the tip of one stack and interact almost only with their first two neighbors.

In the interstellar medium, PAH clusters are not expected to be made of homogenous units but to aggregate PAHs of different sizes whose structures can also be calculated. The stacks in which the larger PAHs occupy inner sites maximize the number of nearest neighbors between carbon atoms and are energetically favored. This segregation property remains for larger clusters made of several stacks, the smallest molecules being located at the tips of the stacks. 


\subsection{Stability in NGC 7023N}

A key question for astrophysics is whether these clusters can survive in regions such as NGC 7023N where they have been proposed to be present. Their stability results from a competition between formation, upon PAHs/clusters collisions, and destruction by the UV flux. The corresponding rates have been estimated from molecular modelling simulations for clusters made of 4 or 13 coronene molecules (Rapacioli et al. 2006). Formation rates were calculated from collision rates between molecules and clusters, which depend on the temperature and density of the cloud, and the probability of sticking at each collision. This last value is calculated from MD simulations using the TB-MM approach combining the TB model of Van Oanh et al. (2002) with a classical intermolecular pair potentials as mentioned above. To describe the evaporation which might follow a UV photon absorption, one needs to compare the rate of energy relaxation either by IR emission or by evaporation of one molecule. Both phenomena can occur on relatively long timescales (at low energy) and real-time simulations of these processes are not possible. However these values can be obtained with statistical approaches, namely the Phase Space Theory for the molecular evaporation (Calvo \& Parneix 2004) with the rigid body model and a microcanonical IR emission model (Joblin et al. 2002) for the IR cooling.

The calculated clustering timescales are typically $10^{4}-8 \times 10^{4}$ years, much longer than the photoevaporation timescales which are below 17 years. Therefore, these clusters should be destroyed by the UV flux much faster than they can be reformed (Rapacioli et al. 2006). Such clusters could not survive in UV irradiated clouds like the PDR NGC 7023N. These results do not however rule out completely the hypothesis of neutral PAH clusters as the size of astronomical PAH-related VSGs might be larger (larger clusters or larger units) and this would increase their stability in PDRs.

\section{5 lonised PAH clusters}

Ionised molecular clusters are more strongly bound than the neutrals. The charge resonance at the origin of this strong stabilisation, completed by polarization, is treated with the DFTB-CI method.

\subsection{Energetics considerations}

We have investigated ionised coronene clusters in non-relaxed stacked geometries (Rapacioli \& Spiegelman 2009). The charge essentially delocalises over the central molecules: the configurations where the charge is carried by the central molecules have much higher weights in the global CI wavefunction than the configurations where the charge is localised on the edge molecules.

Figure 2 displays the dissociation energy of neutral and cationic coronene stacks corresponding to the evaporation of a single molecule from one stack side. In the neutral cluster, the dissociation energy reaches a limit for sizes above 3 units 


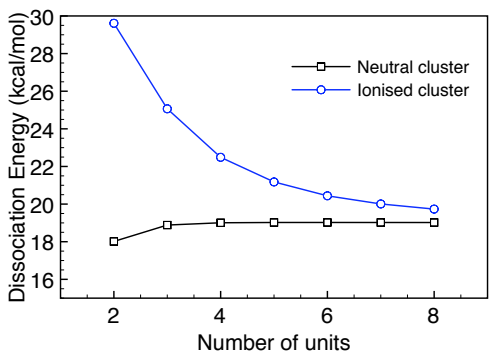

Fig. 2. Dissociation energy of neutral and ionised stacked coronene clusters (Fig. from Rapacioli \& Spiegelman 2009).

as seen previously. The cationic dimer is over 1.5 times more stable than the neutral one. The evaporation rate constant varies approximately exponentially as a function of the dissociation energy, thus cationic clusters will be significantly more resistant to the loss of a neutral molecule. When cluster size increases, the charge distribution is less sensitive to the removal of one unit and the dissociation energy tends to the same limit as observed for neutral clusters.

It should also be noticed that, as the charge delocalisation over the stack stabilises the ionised form, the IP decreases when the cluster size increases. The decrease of the IP with cluster size down to the bulk limit is frequently observed for various families of clusters. For a coronene stack, the IP reaches a limit for clusters above 7-8 units. The IP is then reduced by more than $1 \mathrm{eV}$ compared to that of the isolated molecule. This decrease of the IP could explain the presence of cationic PAH clusters in PDRs regions where isolated PAHs would be neutral. Most of the features will certainly remain valid when achieving full relaxation of the ion geometries. The issues are (i) how large is the core subcluster over which the charge is actually delocalised; (ii) what is the quantitative interplay between geometric relaxation and stability, and the construction of larger clusters; (iii) what is the supramolecular organization of the other units essentially neutral and polarized by this core; and (iv) what are the implications for nucleation and evaporation. Implementation of the analytical gradient in DFTB-CI is underway and should allow to answer precisely these questions.

\subsection{Formation rates}

Although the complete study of ionised PAH cluster formation would require to perform MD to simulate collisions as done for neutral clusters, the evolution of the collision rate can be estimated. Because the VSGs are observed in regions where PAHs are expected to be neutral, it is consistent to focus on collisions between a neutral molecule with a $(P A H)_{n}^{+}$cluster. In such a collision, the cationic cluster induces a dipole on the PAH molecule. The long-range ionic clusters/induced dipole interaction is attractive and scales as $1 / R^{4}$. This interaction is much stronger than in the case of neutral clusters, which scales as $1 / R^{6}$. The formation 
rate can be roughly estimated from a simple Langevin model. The Langevin prediction for the nucleation rate is then given by $k_{n u c l}=2 e(\alpha / \mu)^{1 / 2}$ (see for instance Herbst 2001), where $e$ is the elementary charge, $\alpha$ the polarisability of the PAH, and $\mu$ the reduced mass of the reactants. This leads, for the specific case of NGC $7023 \mathrm{~N}$, to reformation timescales of a few thousands years, much faster than for neutral clusters. The conclusion that small clusters are destroyed faster than they can be reformed could thus be modified for ionised clusters, but a complete study of their stability, including the calculation of the evaporation rates, necessary to answer this question, is under development.

\section{SiPAH complexes}

PAH-related VSGs could contain heteroatoms. The case of Fe is discussed in Simon et al. (this volume). We discuss here SiPAH complexes. The first step in the study of such systems consists in investigating the smallest dimers, that is a single PAH molecule with a single Si atom. Some characteristic features associated to the coordination of Si can already be derived from these simple model systems, in particular concerning the structures and IR spectra of $[\mathrm{SiPAH}]^{+}$complexes.

\subsection{Structures and binding energies}

The structures of $[\mathrm{SiPAH}]^{0 /+}$ complexes have been optimized at the DFT level by Joalland et al. (2009). The SiPAH binding energies in $[\mathrm{SiPAH}]^{+}$complexes were computed to be at least $1.5 \mathrm{eV}$. This value being three times higher than for $[\mathrm{SiPAH}]^{0},[\mathrm{SiPAH}]^{+}$complexes are therefore more likely to survive in the conditions of the ISM than their neutral counterparts. Furthermore, the formation of $[\mathrm{SiPAH}]^{+}$complexes by radiative association is an exothermic process without any expected activation barrier. These $[\mathrm{SiPAH}]^{+}$complexes could then represent a significant fraction of PAH-related species and their presence has been suggested to account for the blue-shift of the $6.2 \mu \mathrm{m}$ band (see also Peeter et al., this volume). The exploration of the PES reveals that in the most stable structures, the Si atom is located on top of a $\mathrm{C}$ atoms on the edge of the PAH molecular structure. It was furthermore demonstrated that the lowest energy path for the motion of the Si atom parallel to the PAH surface is through the edge of the PAH.

\subsection{IR spectroscopy}

\subsubsection{Harmonic}

The IR spectra of such complexes have been calculated with the harmonic approximation at the DFT level. Compared to isolated PAHs, the $[\mathrm{SiPAH}]^{+}$complexes present very soft modes associated to the motion of the Si atom above the PAH surface. For instance, in $\left[\mathrm{SiC}_{10} \mathrm{H}_{8}\right]^{+}$complex, these modes are located at 202, 240, and $413 \mathrm{~cm}^{-1}$. The first two correspond to a Si motion parallel to the PAH plane and have very low intensities. The $413 \mathrm{~cm}^{-1}$ mode corresponds to a Si motion in 
a direction perpendicular to the PAH plane with an intensity of about $12 \%$ of the maximum intensity.

The mid-IR spectra of $[\mathrm{SiPAH}]^{+}$resemble more that of a cationic PAH than that of a neutral PAH. This results from the fact that in the $[\mathrm{SiPAH}]^{+}$complexes, most of the charge is actually carried by the PAH. A significant spectral difference stems from the fact that the symmetry is reduced in the complex. For instance, the $\gamma_{C H}$ band located at $780 \mathrm{~cm}^{-1}$ in $\mathrm{C}_{10} H_{8}^{+}$splits into two subbands at 862 and $786 \mathrm{~cm}^{-1}$ in $\left[\mathrm{Si}_{10} \mathrm{H}_{8}\right]^{+}$. The first band involves mainly the bending of $\mathrm{C}-\mathrm{H}$ bonds from the ring neighboring the Si atom whereas the second $\gamma_{C H}$ band involves the $\mathrm{H}$ atoms coordinated to the other rings and is therefore less perturbed. The $1594 \mathrm{~cm}^{-1}$ band is assigned to a $\nu_{C C}$ mode involving the whole PAH skeleton. It is blue-shifted by $29 \mathrm{~cm}^{-1}$ with respect to that in $C_{10} H_{8}^{+}$. The splitting of the $\gamma_{C H}$ band into two bands and the blue-shift of the $\nu_{C C}$ band are therefore signatures to probe the presence of $[\mathrm{SiPAH}]^{+}$complexes in the ISM.

\subsubsection{Anharmonic}

The spectra calculated at the DFT level are harmonic spectra corresponding to small amplitude motions (spectra at the limit of $\mathrm{T}=0 \mathrm{~K}$ ). In the emission process observed in the ISM, the temperature of such complexes differs from $0 \mathrm{~K}$ and anharmonic effects should be taken into account. In the classical motion approximation, these effects can be derived from MD simulations by computing the Fourier transform of the complex dipole autocorrelation function. The use of DFTB on-the fly allows to perform MD simulations on the required long-timescales (Joalland et al. 2010) and to explore various ranges of temperatures.

For low temperatures, the Si atom remains on top of the same ring. For higher temperatures, there is sufficient energy for the Si atom to overcome the barrier separating two PES minima (differing by the Si position on top of two adjacent cycles) and the average time spent on a given ring decreases as the temperature increases.

Figure 3 shows the evolution of the $\gamma_{C H}$ mode in the naphthalene cation. It exhibits a broadening and a red-shift when the temperature increases. The same behavior is observed in the $\left[\mathrm{SiC}_{10} \mathrm{H}_{8}\right]^{+}$complex for the two $\gamma_{\mathrm{CH}}$ bands that result from the splitting due to the Si coordination. However, the broadening is much more significant for temperatures larger than $600 \mathrm{~K}$, when the system reaches the high temperature mobility regime. At this temperature, the Si oscillation over the two rings is fast enough to couple the two $\mathrm{C}-\mathrm{H}$ modes. The two corresponding bands are thus shifted towards each other, each one displaying a broad wing in the interband region.

The study of complexes with increasing sizes shows that this band broadening can be generalized to the spectra of $[\mathrm{SiPAH}]^{+}$complexes compared to those of the corresponding bare $\mathrm{PAH}^{+}$. Although the spectra calculated for a limited number of SiPAH complexes are not directly able to account the VSG spectrum, this band broadening is consistent with the features observed in the VSG spectrum. 

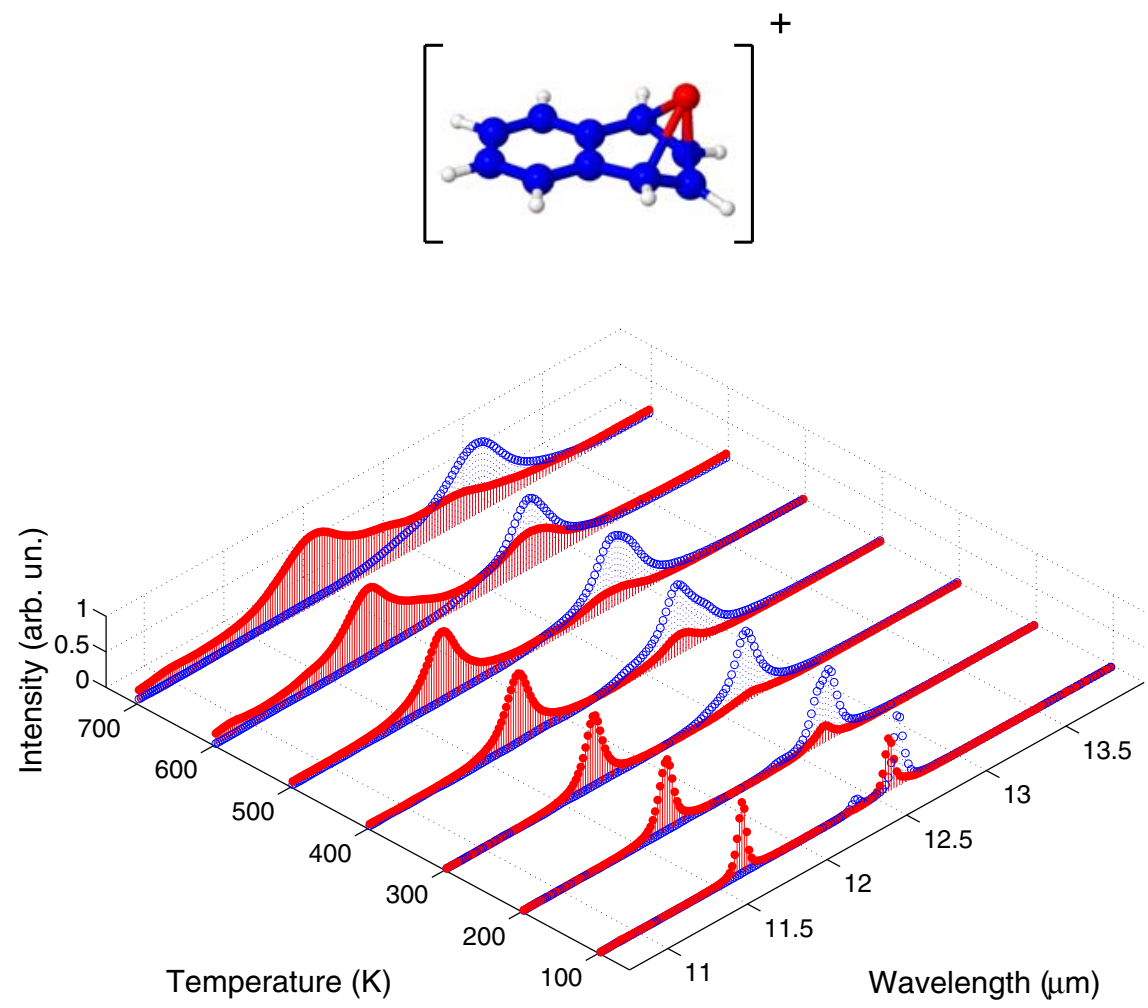

Fig. 3. Evolution of the $\gamma_{C H}$ band in bare $C_{10} H_{8}^{+}$(blue/open circles) and in the $\left[\mathrm{SiC}_{10} \mathrm{H}_{8}\right]^{+}$complex (red/filled circles) as a function of the temperature.

\section{Conclusion}

The spectral evolution of the AIBs in PDRs provides evidence for PAH evolution in molecular clouds. Inside molecular clouds, the spectra present characteristics of small carbonaceous grains which seem to evaporate, where the UV flux becomes strong enough, to give the free-flying PAHs. The theoretical modelling of these grains requires multiscale approaches combining different levels of theory to model the PES.

Possible candidates for these grains are PAH clusters in their neutral form as they are located in regions where PAHs are expected to be neutrals. A global exploration of the structural properties shows that the most stable structures are self assemblies of small units made of PAH stacks. The comparison of calculated nucleation rates for clusters of 4 and 13 coronene molecules versus their evaporation rates in the physical conditions of NGC 7023N leads to the conclusion that such clusters are photoevaporated faster than they can be reformed. 
They are thus not the observed VSGs and other hypotheses must be investigated like for instance clusters made of larger PAHs and/or with more units. Other candidates are cationic PAH clusters. The binding energies are larger than that computed for their neutral counterparts and the long-range attraction of the ionic cluster/polarisable molecule strongly increases the formation rate. Finally we investigated the possibility that VSGs could consist of complexes of PAHs with heteroatoms such as Fe or Si. The harmonic spectra of $[\mathrm{SiPAH}]^{+}$complexes show the appearance of new bands compared to the spectra of bare $\mathrm{PAH}^{+}$. When the temperature increases, these bands are broadened, shifted and mixed to give broad structures. Such effects are in line with the broadening of the AIBs in the VSG spectra.

The question of the VSG nature is still an open question. At the moment, the only conclusion that can be drawn is that these grains are larger than PAHs but strongly related to them. If the small neutral PAH clusters hypothesis has been ruled out, candidates like clusters of larger molecules and/or with more units, ionised PAH clusters and Fe/Si-PAH complexes need further investigations. A global evolution model of PAHs and related species would be very useful to validate/invalidate the various hypotheses (See for instance Montillaud et al., in this volume).

The authors thank F. Calvo and P. Parneix for their contributions in various aspects of the modelling and simulations presented in this overview.

\section{References}

Allamandola, L.J., Tielens, A.G.G.M., \& Barker, J.R., 1985, ApJ, 290, L25

Berné, O., Joblin, C., Deville, Y., et al., 2007, A\&A, 469, 575

Berné, O., Joblin, C., Fuente, A., \& Menard, F., 2009, A\&A, 495, 827

Berné, O., Joblin, C., Rapacioli, M., et al., 2008, A\&A, 479, L41

Calvo, F., \& Parneix, P., 2004, JCP, 120, 2780

Chabbal, S., Stoll, H., Werner, H.-J., \& Leininger, T., 2010, Mol. Phys., submitted

Elstner, M., Hobza, P., Frauenheim, T., Suhai, S., \& Kaxiras, E., 2001, JCP, 114, 5149

Elstner, M., Porezag, D., Jungnickel, G., et al., 1998, Phys. Rev. B, 58, 7260

Fromager, E., \& Jensen, H.J.A., 2008, Phys. Rev. A, 78, 022504

Grimme, S., 2006, J. Comp. Chem., 27, 1787

Herbst, E., 2001, Chem. Soc. Rev., 30, 168

Joalland, B., Rapacioli, M., Simon, A., et al., 2010, JPC A, 114, 5846

Joalland, B., Simon, A., Marsden, C. J., \& Joblin, C., 2009, A\&A, 494, 969

Joblin, C., Szczerba, R., Berne, O., \& Szyszka, C., 2008, A\&A, 490, 189

Joblin, C., Toublanc, D., Boissel, P., \& Tielens, A., 2002, Mol. Phys., 22, 3595

Léger, A., \& Puget, J.L., 1984, A\&A, 137, L5

Oliveira, A., Seifert, G., Heine, T., \& duarte, H., 2009, J. Braz. Chem. Soc., 20, 1193

Podeszwa, R., Bukowski, R., \& Szalewicz, K., 2006, JPC A, 110, 10345 
Porezag, D., Frauenheim, T., Köhler, T., Seifert, G., \& Kaschner, R., 1995, Phys. Rev. B, 51, 12947

Rapacioli, M., Calvo, F., Joblin, C., et al., 2006, A\&A, 460, 519

Rapacioli, M., Calvo, F., Spiegelman, F., Joblin, C., \& Wales, D., 2005, JPC A, 109, 2487

Rapacioli, M., Joblin, C., \& Boissel, P., 2005, A\&A, 429, 193

Rapacioli, M., \& Spiegelman, F., 2009, EpJD, 52, 55

Rapacioli, M., Spiegelman, F., Mirtschink, A., \& Scemama, A., 2010, in preparation

Rapacioli, M., Spiegelman, F., Talbi, D., et al., 2009, JCP, 130, 244304

Rhee, Y.M., Lee, T.J., Gudipati, M.S., Allamandola, L.J., \& Head-Gordon, M., 2007, PNAS, 104, 5274

Seifert, G., Porezag, D., \& Frauenheim, T., 1996, IJQM, 58, 185

Simon, A., \& Joblin, C., 2007, JPC A, 111, 9745

Simon, A., \& Joblin, C., 2009, JPC A, 113, 4878

Simon, A., \& Joblin, C., 2010, ApJ, 712, 69

Simon, A., Joblin, C., Polfer, N., \& Oomens, J., 2008, JPC A, 112, 8551

Stone, A., 1997, The Theory of Intermolecular Forces, 0198558848, (USA, Oxford: University Press)

Toulouse, J., Colonna, F., \& Savin, A., 2004, Phys. Rev. A, 70, 6

Van Oanh, N.T., Parneix, P., \& Bréchignac, P., 2002, JPC A., 106, 10144

Wu, Q., Cheng, S.-L., \& Van Voorhis, T., 2007, JCP, 127, 164119

Zhao, Y., \& Truhlar, D. G., 2008, JPC C, 112, 4061

Zhechkov, L., Heine, T., Patchovskii, S., Seifert, G., \& Duarte, H., 2005, JCTC, 1, 841 